\title{
THE INFLUENCE OF EXPERTISE AND EXPERIMENTAL PARADIGMS ON THE VISUAL BEHAVIOR OF TENNIS ATHLETES IN RETURNING A SERVE
}

\author{
Yen-Nan Lin', Matheus M. Pacheco², Yaw-Feng Lin ${ }^{3}$, Chia-Tso Huang ${ }^{4}$, and Ju-Han Lin ${ }^{5}$ \\ ${ }^{1}$ Department of Life Sciences, National Dong Hwa University, Taiwan \\ ${ }^{2}$ Motor Behavior Laboratory, Department of Kinesiology, The University of Georgia, USA \\ ${ }^{3}$ Department of Physical Education, National Ping Tung University, Taiwan \\ ${ }^{4}$ Pitotech CO., LTD, Changhua, Taiwan \\ ${ }^{5}$ Department of Physical Education and Kinesiology, National Dong Hwa University, Taiwan
}

Original scientific paper

DOI 10.26582/k.53.2.10

\begin{abstract}
:
To return a serve, one must pick up information from the server's kinematics and anticipate the ball trajectory. Although the perceptual requirements are important, the literature diverges in terms of the differences between experts and novices as well as the importance of the experimental paradigm (in-situ vs. video-based) for the results. This study aimed to address both concerns. We compared experts' ( $=7,20.6 \pm 1.1$ years of age) and novices' ( $\mathrm{n}=7,20.0 \pm 0.4$ years of age) visual pattern when returning a serve (Experiment 1) and the influence of the experimental paradigm in experts (Experiment 2). Experts fixated more and longer the upper body and ball, while novices showed a more distributed pattern and with longer fixations outside of the server's body. Also, the pattern was different when comparing in-situ and laboratory settings, differing mainly in fixation frequency. The influence of expertise was observed in qualitative (relative) and quantitative (absolute) measures of visual behavior with the setting having an important influence. Thus, studies should be as close to the actual situation if trying to understand experts' behavior.
\end{abstract}

Key words: visual search, gaze behavior, sports, experts, novices

\section{Introduction}

The ability to perceive and anticipate events in a timely manner is one of the most important abilities in sport. In tennis, this is highly apparent, especially in the situation of returning the serve. Speed of the served tennis ball can achieve velocities up to 250 $\mathrm{km} \cdot \mathrm{h}^{-1}$ for men and $200 \mathrm{~km} \cdot \mathrm{h}^{-1}$ for women. Surprisingly, expert tennis players can respond to such speeds demonstrating their good ability to perceive and anticipate. The literature tends to agree that experts can pick up information from the opponent's movement pattern to anticipate their own response (e.g., Williams, Davids, Burwitz, \& Williams, 1994; Williams, Davids, \& Williams, 1999). The response observed in novices, however, would not be as effective as it is for experts. Many studies have explored whether players' visual behavior in these situations could be the source of difference between experts and novices (e.g., for a review, see Mann, Williams, Ward, \& Janelle, 2007).

The literature on tennis is still sparse and highly centered on laboratory-based designs (e.g.,
Murray \& Hunfalvay, 2017). For instance, Goulet, Bard, and Fleury (1989), using a video-based paradigm, showed that experts performed more fixations than novices during the ritual phase of the serve (focusing more on the areas of shoulder/trunk and head) in comparison to novices - with no large differences in preparation and execution. Nevertheless, the necessary information for predicting where the ball would land between them was different (experts would need only the preparation and initial phase of execution). Ward, Williams, and Bennett (2002), in testing whether perception would occur from relative motion of the body (also using video simulation), observed that experts fixated more around trunk/hip and head/shoulders, while novices showed a focused fixation on hand/ racket and the ball. The only study that investigated visual-search behavior in-situ, Singer et al. (1998) investigated differences between experts of different ranks. They showed that the better ranked players showed a smooth pursuit tracking of the ball after the serve, while lower ranked players showed a higher number of saccades. 
Although some argue that both laboratorybased and in-situ designs have each their problems (ecological validity and control, respectively) (Kredel, Vater, Klostermann, \& Hossner, 2017), the central concern is to understand athletes' capacities in real situations. Thus, it is of concern the potential differences that the video paradigm can invoke in visual search behavior when compared to in-situ observations. Indeed, this has been demonstrated in other sports (e.g., Dicks, Buton, \& Davids, 2010; see also Mann, et al., 2007). Dicks et al. (2010), for instance, demonstrated that the visual pattern of the goalkeeper in the soccer penalty kick is rather focused on the ball in an in-situ condition compared to a video-based one - where the focus on the head, body, and non-dominant leg has been demonstrated. Such a large difference in behavior leads to, first, an erroneous understanding of athletes' capacities and processes and, second, interventions that are designed based on inappropriate findings.

The issue has been addressed in tennis studies (Farrow \& Abernethy, 2003; Farrow, Abernethy, \& Jackson, 2005; see also Reina, Moreno, \& Sanz, 2007). Nevertheless, the comparison was made only in terms of performance (in a temporal occlusion paradigm) not the actual visual search performed. Farrow and Abernethy (2003) showed that, quantitatively, performance for the situations that involved a striking movement response were more accurate than when the athletes were just required to provide verbal responses. However, the pattern of responses between in-situ (with movement responses) and video simulation (with paper and pencil responses) were qualitative the same (Farrow, et al., 2005).

Theoretically, variation in how information is presented and which actions are required could elicit a number of modifications in the movement patterns and information attended. From a dynamical systems point of view, the constraints of the task (rules, goals) and environment (how the setting is formulated and changing) would channel the behavior differently (Newell, 1986) - even if one is to control for the individuals being compared (experts and novices). On the one hand, then, one would expect large disparities of behavior between different experimental designs. On the other hand, a given perception-action regime can be maintained even when such constraints are slightly varied (see Kelso, 1995). The tennis literature on visual search on the return of serve is still highly based on the video simulation paradigm. This might provide erroneous conclusions on the actual search behavior and limit understanding of experts' behavior and how changes occur from novices to experts in this context. Thus, the literature requires more information about the actual visual search behavior in-situ in tennis, comparing experts and novices, and a comparison between video-based and in-situ behavior. This is what the present study did.

\section{Experiment 1}

In this first experiment, we compared experts and novices' visual-search behavior in a real situation (in-situ) requiring them to perform the serve return.

\section{Methods}

\section{Participants}

Fourteen male college-level tennis players were recruited to participate in the experiment. Seven participants were classified as experts $(20.6 \pm 1.1$ years of age; 11.21 years of tennis experience; part of the College Tennis Team ranked $4^{\text {th }}$ at the 2016 National Intercollegiate Athletic Games) and seven participants were classified as novices $(20.0 \pm 0.4$ years of age; $1 \pm 0.5$ years of tennis experience). All participants stated having normal vision and reported having, at the time, no injury that would prevent them from participating in regular tennis matches. All participants signed an informed consent form and the study was approved by the Mennonite Christian Hospital Institutional Review Board.

\section{Material and apparatus}

The visual behavior was captured by the portable eye movement instrument, Mobile Eye ASL Mexg (EST, Germany) that used the corneal reflex for monocular tracking. The device captures and stores data on a hard drive that is used for later assessment. The sampling frequency was set at 30 $\mathrm{Hz}$ and the accuracy of the device was within 0.5 degrees of visual angle. The number of fixation points, fixation time and frequency were assessed using Mangold Interact software (Mangold International, Canada). In addition, a radar gun, JUGS (R-1000), was used to measure velocity of the serve. The gun was set in front of the server at $1.75-\mathrm{m}$ height and 1-m distance.

Fixation locations. In Ward et al. (2002), eight fixation locations of the server's body were used to describe the visual behavior of novices and experts in receiving a serve when a video was shown to the participants: trunk/hips, arm/hand, leg/foot, racket, ball, racket-ball contact, unclassified. Nevertheless, in the present study, we identified that the instrument was unable to differentiate between these on the actual court. The experimenters and two coaches (10 years of experience) defined four main regions that would influence the behavior based on their experience: (1) upper body (includes chest, arm, and racket), (2) lower body (pelvis and lower limbs), (3) the ball, (4) background (outside of the body of the server, excluding the sphere). 


\section{Procedures}

At the arrival on the court, researchers stated the purpose of the study to the participant and then calibrated the eye-tracking device. The participant was then instructed to prepare to return the ball served from the deuce part of the court. He practiced the return with the device for three serves for familiarization. For the actual experimental trials, the participant returned 10 serves that were randomly ordered to occur five times at the left or right side of the participant. After receiving the ball, the participant waited for the signal of the experimenter indicating that the ball was served within the required speed range (130 to $\left.150 \mathrm{~km} \cdot \mathrm{h}^{-1}\right)$ and the landing point was correct - a successful trial. This speed range was chosen because it represents an average serve speed at the collegiate level. Then, whenever the trial was not successfully executed, it was repeated at the end (this did not occur often). The eye-tracking device stopped collecting data at the moment the ball passed over the net after the return strike (each trial took around $6.021 \pm 1.676$ seconds).

\section{Data analysis}

The main measures of the study were fixation frequency and time in different fixation locations of the server's body as a function of expertise (groups) and stage of the serve movement. The fixation was defined as a period of 100 miliseconds (three frames) in which the eye maintained the location within 3 degrees (Panchuk \& Vickers, 2006). Total fixation time (and frequency) can vary depending on each individual's visual search pattern. This can result in a group showing more fixation times in all regions even when the pattern of fixation is not different. Thus, we also provided a relative comparison (in terms of percentage) to provide the information whether the overall pattern in fixation time and frequency differed between the groups. In this way, we can differentiate quantitative from qualitative differences between the groups (and conditions [see Experiment 2]).

Given the non-normality of distribution for some measures, we used robust analyses (Wilcox, 2017). Thus, we used robust two-way ANOVAs for (each) fixation time and frequency as dependent variable with the fixation location as a repeated measure and expertise as a between-group measure ( 2 groups $\mathrm{x} 4$ locations). When the interaction was significant, we only reported the interaction results. Additionally, we provided the effect size in terms of Algina, Keselman, and Penfield (2005) measure of effect size $(\delta)$ for the ANOVA effects (each significant post-hoc comparison). This measure, $\delta$, is similar to Cohen's $d$ for paired comparisons but applies trimmed means and Winsorized variances.
We also compared the performance, speed of serve, total time and number of fixations (total frequency) between the groups using the Yuen's Method for comparing trimmed means. For the pairwise tests, we used the explanatory measure of effect size $(\xi)$.

\section{Results}

\section{Performance and speed of serve}

In terms of performance, we found that experts showed an average of seven successful returns (the return landed inside of the opponent's court), 2.71 returns that landed outside of the opponent's court and 0.28 aces from the server. Novices showed an average of 1.57 successful returns, 6.57 returns that landed outside of the opponent's court and 2.14 aces from the server. Considering the sum of returns that landed inside of the court as the performance variable, the Yuen test for independent samples revealed that, as expected, experts performed better than novices, $T_{y}(4)=10.36, \mathrm{p}<.001, \xi=0.88$.

The average speed of serve for experts was slightly higher $\left(141.94 \mathrm{~km} \cdot \mathrm{h}^{-1}\right)$ than it was for novices $\left(139.34 \mathrm{~km} \cdot \mathrm{h}^{-1}\right)$. The Yuen test for independent samples showed that this was also significant $\left(T_{y}(7.91)=4.93, \mathrm{p}=.001, \xi=0.79\right)$.

\section{Fixation time}

Table 1 shows the mean, standard deviation, and percentage for each group in terms of each location.

Absolute. Figure 1 shows the fixation time and fixation frequency for each location. The robust ANOVA revealed a non-significant main effect of groups $(\mathrm{p}=.158)$ but a significant main effect of location, $Q=42.65, \mathrm{p}<.001$, and an interaction effect between the group and location, $Q=50.35, \mathrm{p}<.001$. The post-hoc analyses showed that the expert group spent more time fixating the ball $(\mathrm{p}<.001$, $\delta=4.87$ ) than novices. Also, novices spent more time in fixating other locations $(\mathrm{p}=.006, \delta=3.19)$ than experts.

Within each group, the post-hoc analyses showed that experts spent more time fixating the upper body than the lower body $(\mathrm{p}<.001, \delta=3.29)$ and other locations $(\mathrm{p}<.001 ; \delta=2.16)$; then less time fixating the lower body than the ball $(\mathrm{p}<.001$, $\delta=10.62)$; and more time the ball than other locations $(\mathrm{p}<.001, \delta=4.32)$. Also, the analyses showed that novices spent more time fixating other locations than the lower body ( $\mathrm{p}=.002, \delta=2.78)$.

Relative. Figure 2 shows the relative fixation time and fixation frequency for each location. The group differences in the fixation time showed to be non-significant. Experts showed similar fixation times as novices $(p=.123)$. Thus, we did not compare the fixation time between the groups considering the time relative to the total spent fixating each location. 
Table 1. Mean \pm standard deviation (mean percentage) of fixation time and fixation frequency between experts and novices

\begin{tabular}{llcccc}
\hline Groups & & Upper body & Lower body & Ball & Other \\
\hline \multirow{3}{*}{$\begin{array}{l}\text { Experts } \\
(n=7)\end{array}$} & Fixation time (s) & $\begin{array}{c}1.54 \pm 0.45 \\
(\%)\end{array}$ & $\begin{array}{c}0.14 \pm 0.17 \\
(3 \%)\end{array}$ & $\begin{array}{c}1.39 \pm 0.21 \\
(44 \%)\end{array}$ & $\begin{array}{c}0.13 \pm 0.08 \\
(4 \%)\end{array}$ \\
\cline { 2 - 5 } & Fixation frequency (count) & $\begin{array}{c}3.97 \pm 0.65 \\
(49 \%)\end{array}$ & $\begin{array}{c}0.47 \pm 0.41 \\
(5 \%)\end{array}$ & $\begin{array}{c}3.00 \pm 0.40 \\
(37 \%)\end{array}$ & $\begin{array}{c}0.61 \pm 0.38 \\
(7 \%)\end{array}$ \\
\hline & $(\%)$ & & & & \\
\hline & & $0.76 \pm 0.38$ & $0.41 \pm 0.21$ & $0.28 \pm 0.13$ & $0.94 \pm 0.29$ \\
Novices & Fixation time (s) & $(30 \%)$ & $(16 \%)$ & $(13 \%)$ & $(39 \%)$ \\
$(n=7)$ & $(\%)$ & $1.45 \pm 0.33$ & $1.25 \pm 0.22$ & $0.42 \pm 0.19$ & $4.01 \pm 0.37$ \\
& Fixation frequency (count) & $(20 \%)$ & $(17 \%)$ & $(5 \%)$ & $(56 \%)$ \\
\hline
\end{tabular}
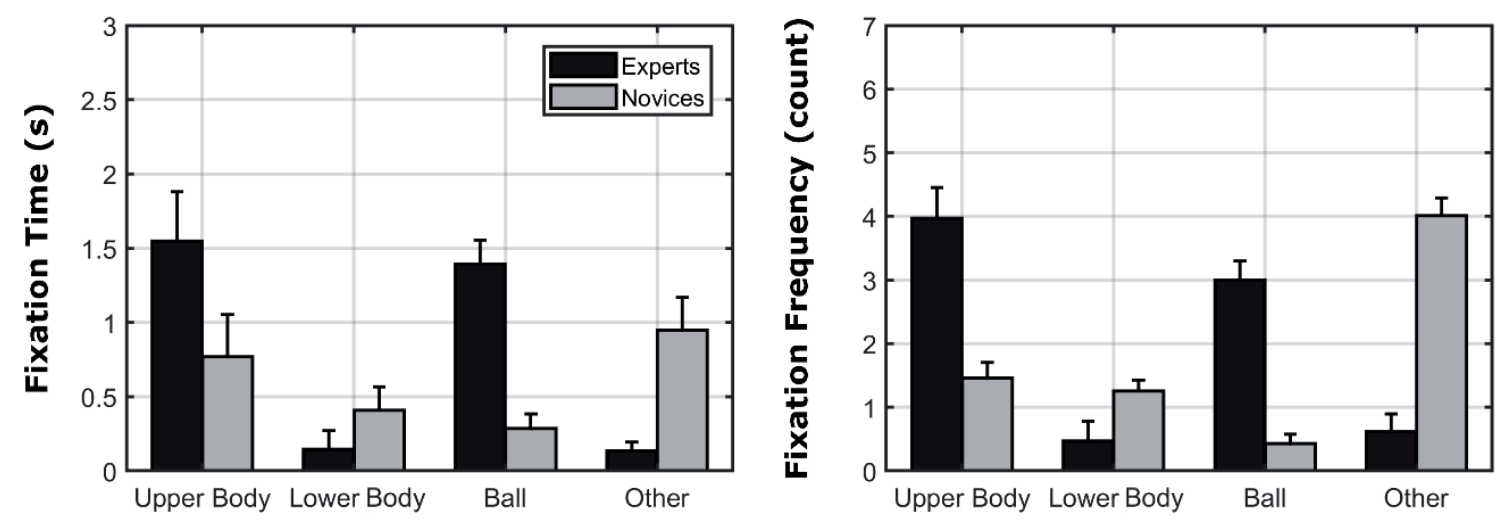

\section{Locations}

Figure 1. Mean (bar) and 95\% confidence interval (error bars) of the absolute fixation time (left) and fixation frequency (right) for experts (black) and novices (gray) per location.
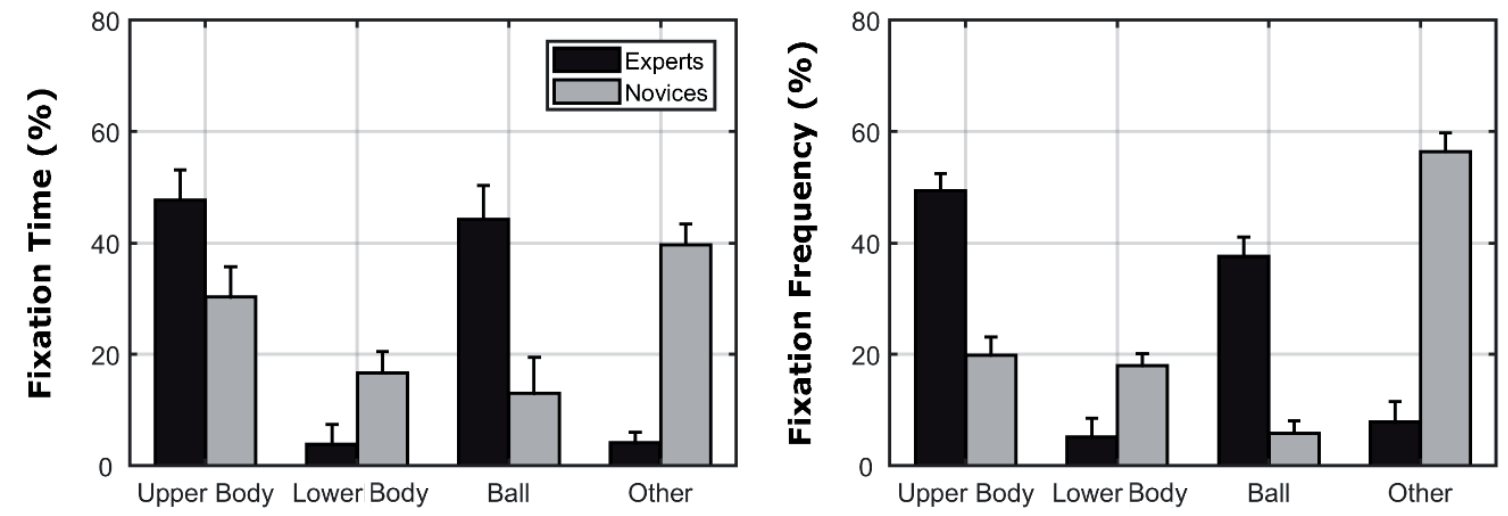

\section{Locations}

Figure 2. Mean (bar) and 95\% confidence interval (error bars) of the relative fixation time (left) and fixation frequency (right) for experts (black) and novices (gray) per location.

\section{Fixation frequency}

Absolute. The robust ANOVA revealed a non-significant main effect for group $(\mathrm{p}=.154)$ but a significant main effect for location, $Q=41.08$, $\mathrm{p}<.001$, and an interaction effect between group and location, $Q=207.13, \mathrm{p}<.001$. The post-hoc analyses showed that experts showed higher fixation frequency on the upper body $(\mathrm{p}<.001, \delta=3.27)$ and the ball $(\mathrm{p}<.001, \delta=6.23)$ than novices. Novices showed higher fixation frequency on other locations $(\mathrm{p}<.001, \delta=6.56)$ than experts.

Within each group, the post-hoc analyses showed that experts fixated more frequently the upper body than the lower body $(\mathrm{p}<.001, \delta=4.97)$ and other locations $(\mathrm{p}<.001, \delta=4.54)$, less frequently the lower body than the ball $(\mathrm{p}<.001, \delta=17.28)$; and more frequently the ball than other locations $(\mathrm{p}<.001, \delta=2.46)$. Also, the analyses showed that 
novices fixated more frequently the lower body than the ball $(\mathrm{p}<.001, \delta=2.15)$, and more frequently other locations than all the other parts (the upper body: $\mathrm{p}<.001, \delta=3.81$; the lower body: $\mathrm{p}<.001, \delta=4.75$; the ball: $\mathrm{p}<.001, \delta=7.94)$.

Relative. The group differences in the fixation frequency showed to be significant $\left(T_{v}(5.12)=2.67\right.$, $\mathrm{p}=.043, \xi=0.73)$. Experts showed higher fixation time than novices $(0.22 \pm .09)$. Thus, we compared the fixation frequency between the groups considering the frequency relative to the total frequency spent on each location.

The robust ANOVA revealed a non-significant effect of group $(p=.584)$ but significant main effects of location, $Q=38.61, \mathrm{p}<.001$, and an interaction effect between group and location, $Q=490.00$, $\mathrm{p}<.001$. The post-hoc analyses showed that experts showed higher fixation frequency on the upper body $(\mathrm{p}<.001, \delta=7.05)$ and the ball $(\mathrm{p}<.001, \delta=12.28)$ than novices. Novices showed higher fixation frequency on the lower body $(\mathrm{p}<0.001, \delta=2.33)$ and other location $(\mathrm{p}<.001, \delta=8.19)$ than experts.

Within each group, the post-hoc analyses showed that experts fixated more frequently the upper body than all the other parts of the body (the lower body: $\mathrm{p}<.001, \delta=5.15$; the ball: $\mathrm{p}<.001, \delta=2.48$; other locations: $\mathrm{p}<.001, \delta=6.79)$, less frequently the lower body than the ball $(\mathrm{p}<.001, \delta=5.67)$; and more frequently the ball than other locations $(\mathrm{p}<.001$, $\delta=3.64$ ). Also, the analyses showed that novices fixated more frequently the lower body than the ball $(\mathrm{p}<.001, \delta=2.18)$, and more frequently other locations than all the other parts (the upper body: $\mathrm{p}<.001, \delta=3.73$; the lower body: $\mathrm{p}<.001, \delta=5.64$; the ball: $\mathrm{p}<.001, \delta=10.46$ ).

\section{Discussion}

The goal of this first experiment was to investigate the differences between experts and novices' visual search behavior in preparing to return a serve. Experts' visual search behavior was highly centered on the upper body and the ball, while novices shared their fixation time and frequency more equally among other parts of the body. Nevertheless, novices also showed a large amount of time and frequency outside of the server's body ("other" location).

These results are in consonance with the expectation that experts would show a more focused pattern in the visual search than novices (e.g., Murray \& Hunfalvay, 2017; Ripoll, Kerlizin, Stein \& Reine, 1995; Williams \& Elliott, 1999). This contributes to the current idea that experts know from which part of the kinematics they can extract important information about the serve, while novices seem to be unaware of it, being more dispersed (Abernethy, Gill, Parks, \& Packer, 2001; Abernethy \& Zawi, 2007). As observed, experts fixated more frequently the upper body than the ball itself, showing a visual pattern based on the kinematics of the opponent. The overall pattern of fixation differed between the groups - even when the total time and frequency was controlled. While the expert group was highly focused on the upper body and the ball (more than $90 \%$ of time and frequency), the novices shared similar percentages for the upper and lower body.

Ward et al. (2002), nevertheless, showed that the novices spent more time fixating the area of racket/ ball and less the trunk-hip region than the experts. Additionally, they showed a more distributed pattern of search for experts than in our study. The differences might have emerged from the fact that Ward et al. (2002) used the video simulation paradigm. Dicks et al. (2010) showed a more focused pattern for expert goalkeepers in an in-situ context when compared to a video simulation paradigm. This is probably the case for our more focused pattern of experts.

Also, we found a high occurrence of fixation outside the body and the ball (other location) in the novice group. In Ward et al. (2002), the novices showed a slightly higher percentage on "unclassified" regions of the vision space, but this was not significant. We believe that the main reason comes from the fact that the environment, server, and the individual's location matter for a response in an in-situ context. In the video simulation paradigm, location relative to the server might not matter much for experts or novices. In the in-situ context, experts are aware - before the serve - where they should stay and might be aware that the server location might be less reliable as a source of information about the serving than the kinematics of the body. Novices might explore other aspects of the scene, which includes the background scenario.

Another possibility comes from Singer et al. (1998). Lower rank individuals showed more saccades between the ball and expected landing region during the serve, while better players preferred to pursue the ball during all times. Assuming that the number of saccades between the ball, landing region, server, individual's own location, etc. increases as the rank becomes lower, it is possible that novices overuse such a strategy. This might be even more present in conditions that the player is required to return the serve (in-situ).

\section{Experiment 2}

The literature on visual behavior claims that the form of presentation (in-situ, video, etc.) alters the visual behavior and, thus, any presentation that deviates from the real situation of the game might provide unreliable results (Dicks, et al., 2010; Mann, et al., 2007, but see Kredel, et al., 2017). This does not need to be the case for all sports. Farrow et al. (2005) showed that, in tennis, the results in occlusion paradigms were similar between in-situ and videobased situations. The fact that the video-based para- 
digm might reproduce the real context have practical relevance in the sense that training and evaluation can be done to assess perceptual abilities or strategies from athletes in simpler settings (e.g., Williams, Ward, Knowles, \& Smeeton, 2002) with more appropriate techniques and control (Kredel, et al., 2017). In the present experiment, we tested whether experts would change their visual behavior when comparing in-situ and video-based situations.

\section{Methods}

\section{Participants}

Seven male college-level tennis players were recruited to participate in the experiment (none of them participated in the first experiment). All of them were considered experts $(20.6 \pm 1.1$ years of age; $11.2 \pm 1.1$ years of experience; part of the College Tennis Team ranked 4th at the 2016 National Intercollegiate Athletic Games). All participants stated having normal vision and signed an informed consent form. The study was approved by the Mennonite Christian Hospital Institutional Review Board.

\section{Materials and apparatus}

All material and apparatus were the same as in Experiment 1.

\section{Procedure}

At the arrival at the site (laboratory or court), researchers stated the purpose of the study to the participant and then calibrated the eye-tracking device. For the in-situ condition, participants performed the same procedure described in Experiment 1 . For the video condition, participants stood 4-m away of a projection screen (1.8 $\mathrm{m}$ height $\mathrm{x} 2$ $\mathrm{m}$ width). The same player used for serving was filmed performing 10 serves - randomly ordered with five being to the right side of the deuce part of the court and five to the left. As in the in-situ condition, participants prepared as if they were to receive and return the ball and practiced the same three trials for familiarization. Then, they performed 10 trials of "returning the serve" by moving to the direction anticipated and performing the striking motion. For both situations, the eye-tracking device stopped recording when the ball passed over the net.

\section{Data analysis}

The measures and analyses followed the ones in Experiment 1. The only differences were that there were between-subjects independent variables (no groups) and there is condition (in-situ and video-based) as the second repeated measure variable for robust ANOVA (thus, two two-condition $\mathrm{x}$ four body location robust repeated measures ANOVAs for fixation time and frequency). Also, to compare total time and frequency, we used the Yuen dependent sample test. The measure of effect size was the explanatory measure of effect size $(\xi)$.

\section{Results}

Table 2 shows the mean, standard deviation, and percentage for each condition in terms of each location.

\section{Fixation time}

Absolute. Figure 3 shows the absolute fixation time and fixation frequency for different locations and conditions. The robust ANOVA revealed significant main effects of condition, $Q=87.63$, $\mathrm{p}<.001$, location, $Q=69.02, \mathrm{p}<.001$, and an interaction effect between condition and location, $Q=83.95$, $\mathrm{p}<.001$. The post-hoc analyses showed that in the video condition, the individuals spent more time fixating the upper body $(\mathrm{p}<.001, \xi=0.86)$ and the ball $(\mathrm{p}=.024, \xi=0.85)$ than in the in-situ condition.

Within each condition, the post-hoc analyses showed that in the video condition, experts spent more time fixating the upper body than the lower body $(\mathrm{p}<.001, \xi=5.87)$ and other locations $(\mathrm{p}<.001$, $\xi=7.48$ ), as well as more the ball than the lower body $(\mathrm{p}<.001, \xi=2.35)$. Also, the analyses showed that in the in-situ condition, experts fixated more time the upper body than the lower body ( $\mathrm{p}=.004, \xi=2.31$ ) and other location ( $\mathrm{p}=.003, \xi=2.45)$; and more time the ball than other location ( $\mathrm{p}=.007 ; \xi=2.02$ ).

Relative. The condition differences in the fixation time showed to be significant. Experts showed higher fixation time in the video than in the in-situ paradigm $\left(T_{y}(4)=5.11, \mathrm{p}=.006, \xi=0.99\right)$. Thus, we

Table 2. Mean \pm standard deviation (mean percentage) of fixation time and fixation frequency between video and non-video conditions

\begin{tabular}{|c|c|c|c|c|c|}
\hline Condition & & Upper body & Lower body & Ball & Other \\
\hline \multirow{2}{*}{ Video } & $\begin{array}{l}\text { Fixation time (s) } \\
(\%)\end{array}$ & $\begin{array}{c}2.88 \pm 0.34 \\
(52 \%)\end{array}$ & $\begin{array}{c}0.13 \pm 0.29 \\
(2 \%)\end{array}$ & $\begin{array}{c}1.92 \pm 0.43 \\
(35 \%)\end{array}$ & $\begin{array}{c}0.57 \pm 0.52 \\
(9 \%)\end{array}$ \\
\hline & $\begin{array}{l}\text { Fixation frequency (count) } \\
(\%)\end{array}$ & $\begin{array}{c}4.02 \pm 1.72 \\
(40 \%)\end{array}$ & $\begin{array}{c}0.27 \pm 0.47 \\
(2 \%)\end{array}$ & $\begin{array}{c}3.94 \pm 0.72 \\
(43 \%)\end{array}$ & $\begin{array}{c}1.52 \pm 1.06 \\
(13 \%)\end{array}$ \\
\hline \multirow{2}{*}{ In-situ } & $\begin{array}{l}\text { Fixation time }(\mathrm{s}) \\
(\%)\end{array}$ & $\begin{array}{c}1.63 \pm 0.52 \\
(53 \%)\end{array}$ & $\begin{array}{c}0.11 \pm 0.13 \\
(3 \%)\end{array}$ & $\begin{array}{c}1.06 \pm 0.38 \\
(36 \%)\end{array}$ & $\begin{array}{c}0.19 \pm 0.11 \\
(6 \%)\end{array}$ \\
\hline & $\begin{array}{l}\text { Fixation frequency (count) } \\
(\%)\end{array}$ & $\begin{array}{l}4.20 \pm 0.57 \\
(51 \%)\end{array}$ & $\begin{array}{c}0.25 \pm 0.24 \\
(2 \%)\end{array}$ & $\begin{array}{l}2.92 \pm 0.49 \\
(35 \%)\end{array}$ & $\begin{array}{c}0.80 \pm 0.25 \\
(9 \%)\end{array}$ \\
\hline
\end{tabular}




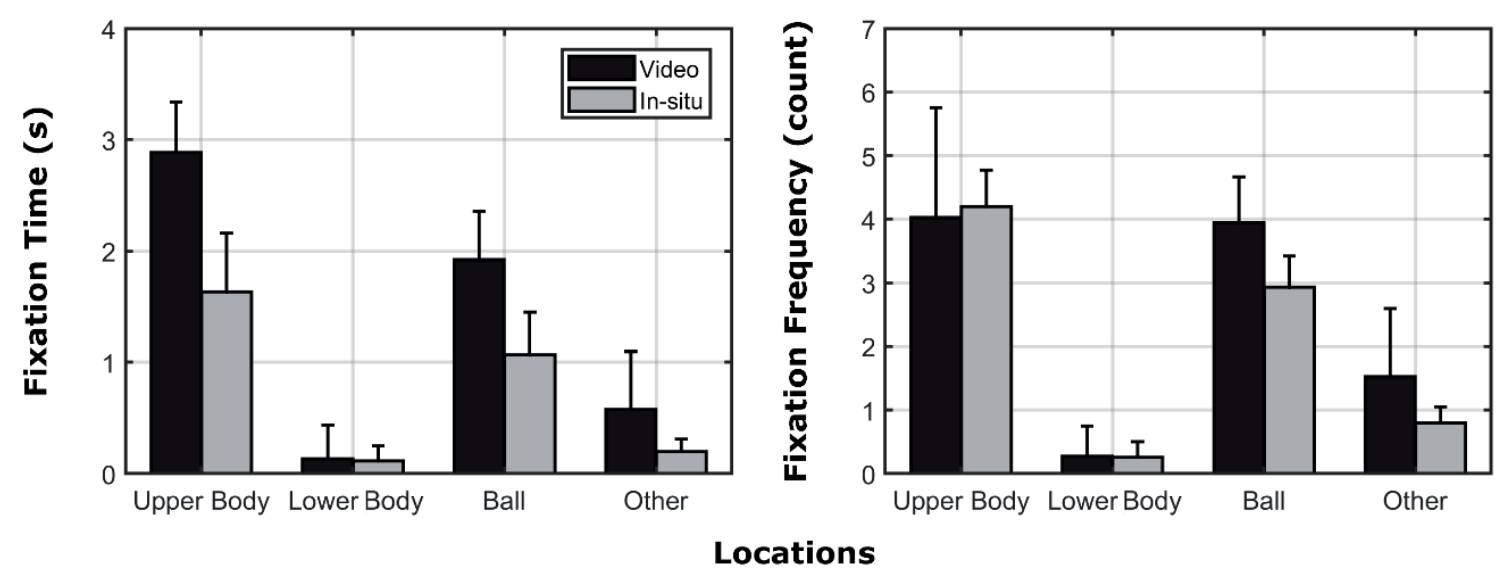

Figure 3. Mean (bar) and 95\% confidence interval (error bars) of the absolute fixation time (left) and fixation frequency (right) in video-based (black) and in-situ (gray) paradigms.
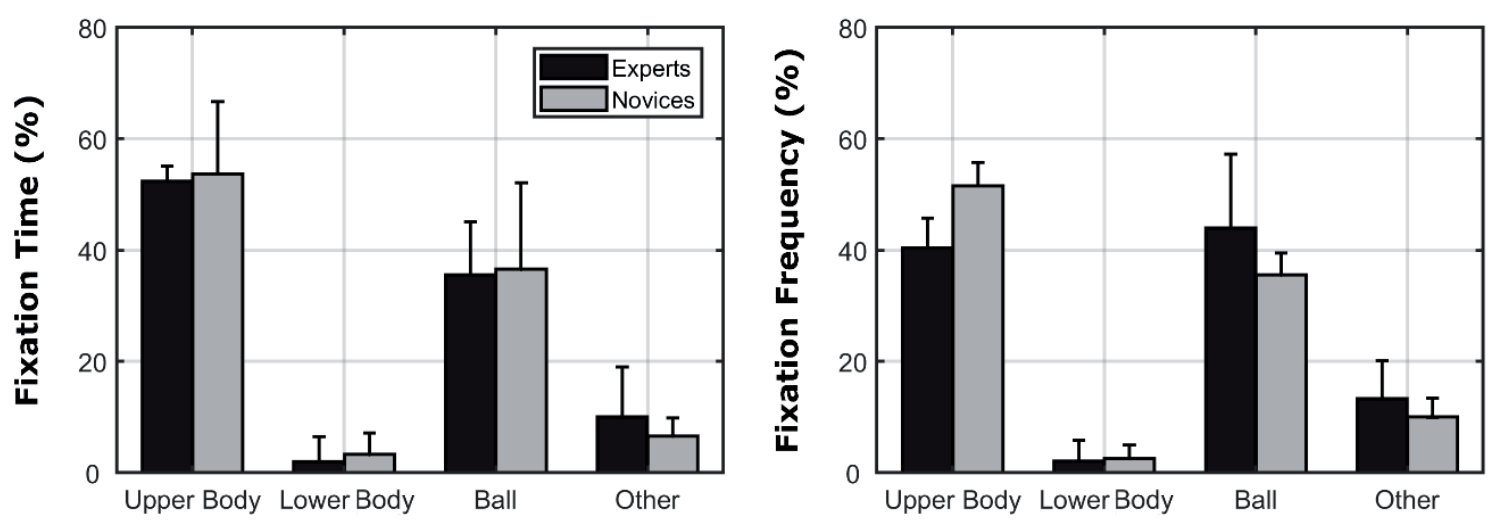

Locations

Figure 4. Mean (bar) and 95\% confidence interval (error bars) of the relative fixation time (left) and fixation frequency (right) in video-based (black) and in-situ (gray) paradigms.

compared the fixation time between conditions considering the time relative to the total spent in each location.

Figure 4 shows the relative fixation time and fixation frequency for different locations and conditions. The ANOVA revealed a non-significant main effect of condition ( $p=.386$ ) but a main effect of location, $Q=221.11, \mathrm{p}<.001$. The interaction effect was also non-significant $(\mathrm{p}=.619)$. The post-hoc analyses showed that experts spent more time fixating the upper body than the lower body $(\mathrm{p}<.001, \xi=5.99)$ and other locations $(\mathrm{p}<.001, \xi=4.06)$.

\section{Fixation frequency}

Absolute. The robust ANOVA revealed a nonsignificant main effect of condition $(\mathrm{p}=.317)$ but a significant main effect of location, $Q=177.59$, $\mathrm{p}<.001, \eta_{\mathrm{p}}{ }^{2}=0.96$, and a significant interaction effect, $Q=9.59, \mathrm{p}<.001$. The post $h o c$ analyses showed no differences between the video and in-situ condition considering each body location.

Within each condition, the post-hoc analyses showed that experts, in the video condition, showed a higher fixation frequency on the ball than on the lower body ( $\mathrm{p}=.001, \xi=3.44)$ and on other location $(\mathrm{p}=.004, \xi=2.45)$. In the in-situ, experts showed a higher fixation frequency on the upper body than on the lower body $(\mathrm{p}<.001, \xi=6.47)$ and on other locations $(\mathrm{p}<.001, \xi=5.11)$, and a higher fixation frequency on the ball than on the lower body $(\mathrm{p}<.001, \xi=12.47)$ and on other locations $(\mathrm{p}<.001$, $\xi=2.54)$.

Relative. There was no difference in total frequency between the video and in-situ conditions $(\mathrm{p}=.370)$. Thus, the relative measure analyses were not performed.

\section{Discussion and conclusions}

The goal of the second experiment was to understand whether differential visual patterns would be observed when video and in-situ conditions are compared. The question followed the contradictory findings that emerged in the literature concerning the differences between in-situ and video paradigms (e.g., Dicks, et al., 2010; Farrow, et al., 2005). Our results revealed that, in general, expert participants perform a qualitatively (not quantitatively) similar pattern of observation in fixation time of serving 
(no relative frequency differences) but a qualitative different pattern considering fixation frequency.

The dissimilarity between video and in-situ conditions contradicts Farrow et al. (2005) who showed that the occlusion paradigm (either progressive or moving window modes) revealed similar patterns of results when anticipation was measured. We found that experts showed longer fixation times in the video paradigm than in the in-situ condition. A comparison with the results of the Experiment 1 shows that the in-situ condition for experts in both experiments showed similar numbers - which reinforces the difference. The differences between paradigms seem to point to a "less conservative" pattern in the video paradigm. Under the video paradigm, every moment that a location is focused on, the focus is maintained for a while. This probably occurs because the time constraints on the performer are softened given that there is no requirement for the response to match the timing of the ball being served. This would also allow an increase in fixations (with longer times) on other regions (similar to Dicks, et al., 2010). Additionally, the differences in fixation frequency also highlight the fact that the video paradigm is less constrained - the in-situ condition led to more directed fixations.

The present study aimed at two main goals: to provide an in-situ comparison between experts and novices in the visual pattern of the player returning the serve in tennis; and to provide a comparison between video and in-situ conditions in the visual pattern in experts. The general outcomes were that experts showed more fixations and longer fixation times than novices being more focused on the upper body and the ball, while novices shared similar frequencies of focus on the upper body, the lower body and the ball. Novices spent longer times fixating outside of the server's body. In addition, experts seem to maintain a general pattern in the visual search between video and in-situ paradigms - with small quantitative differences between both.

The current results seem to follow, in general, the results of other studies in that experts showed a more restricted visual pattern than novices (Ripoll, et al., 1995; Williams and Elliott, 1999). The fact that novices showed a more shared percentage and a decreased fixation time (which might reflect more saccades in less time) corroborates the idea that experts pick up information from relevant cues with no necessity to search for information in the same way novices might do.

Piras, Pierantozzi, and Squatrito (2014), in an in-situ study, showed that novice judo athletes focused more on the sleeves - contrary to experts that had a more central focus (e.g., face and lapel). They (and Ward, et al., 2002) showed that, independent of the paradigm, the novices focused on the "action-related" region. We did not find this overemphasis on the "action-related" region for novices.
It is possible that the changes in visual pattern for novices occur as a consequence of where the action-related region is when video and in-situ conditions are compared. In the video context, the arm and racket are the most related to action region and thus novices might focus on this area. Nevertheless, in the in-situ condition, one can argue that the most proximal region for action is the landing area. This would make novices change the observed visual pattern provided a change in the paradigm from racket to the ball landing location ("other locations" in our study). This would explain the differences between our results and those of Ward et al. (2002) and still corroborate the idea that novices are focusing on the more action-related region of the scene (Piras, et al., 2014; Ward, et al., 2002).

A limitation in the current paper is the number of participants. This arises from the difficulty to collect data in-situ using the eye tracking technology. Beyond the difficulties of bringing and manipulating the equipment outside of the lab, there is the problem of recalibrating if the motion of the participant also causes changes in the position of the equipment (see Singer, et al.,1998). Nevertheless, we observed that the effect size of most comparisons overcame such difficulty to illustrate an overall difference between novices and experts and revealing their preferred regions of fixation. We argue that our study revealed general and consistent patterns of visual search behavior in the return of serve that provide initial ground for further testing. Thus, although the statistical inference methods might have suffered from this limitation, we are convinced that these results are valuable for researchers and practitioners.

In summary, the two primary aims of this study were to compare experts and novices in the in-situ setting and investigate the changes that occur when different experimental paradigms are employed (in-situ vs. video). We found that experts show a more consistent visual pattern, mainly focusing on the upper body and the ball, while novices presented a more distributed pattern. Also, when a video paradigm was implemented, we saw a less concise pattern in experts.

Despite the methodological tendency of the questions posed in the present manuscript, we believe that our results also provide directions in terms of applications. Our results demonstrate that in a real situation, experts are focused on the upper part of the body and on the ball when the opponent is preparing to serve. We interpreted this as a tendency for experts to try to extract kinematic patterns from these parts. Then, it might be that in training interventions, coaches and practitioners should attempt to direct players' attention to features at these areas - and these might be sufficient to predict the opponent' intentions. 


\section{References}

Abernethy, B., Gill, D.P., Parks, S.L., \& Packer, S.T. (2001). Expertise and the perception of kinematic and situational probability information. Perception, 30, 233-252. doi: 10.1068/p2872

Abernethy, B., \& Zawi, K. (2007). Pickup of essential kinematics underpins expert perception of movement patterns. Journal of Motor Behavior, 39, 353-367. doi: 10.3200/JMBR.39.5.353-368

Algina, J., Keselman, H.J., \& Penfield, R.D. (2005). An alternative to Cohen's standardized mean difference effect size: A robust parameter and confidence interval in the two independent groups case. Psychological Methods, 10, 317-328.

Dicks, M., Button, C., \& Davids, K. (2010). Examination of gaze behaviors under in situ and video simulation task constraints reveals differences in information pickup for perception and action. Attention, Perception, and Psychophysics, 72, 706-720. doi: 10.3758/APP.72.3.706

Farrow, D., \& Abernethy, B. (2003). Do expertise and the degree of perception-action coupling affect natural anticipatory performance? Perception, 32, 1127-1139. doi: 10.1068/p3323

Farrow, D., Abernethy, B., \& Jackson, R.C. (2005). Probing expert anticipation with the temporal occlusion paradigm: Experimental investigations of some methodological issues. Motor Control, 9, 332-351. doi: 10.1123/mcj.9.3.330

Goulet, C., Bard, C., \& Fleury, M. (1989). Expertise differences in preparing to return a tennis serve: A visual information processing approach. Journal of Sport and Exercise Psychology, 11, 382-398. doi: 10.1123/jsep.11.4.382

Kelso, J.A.S. (1995). Dynamic patterns. Cambridge, MA: MIT Press.

Kredel, R., Vater, C., Klostermann, A., \& Hossner, E.-J. (2017). Eye-tracking technology and the dynamics of natural gaze behavior in sports: A systematic review of 40 years of research. Frontiers in Psychology, 8, 1845. doi: 10.3389/fpsyg.2017.01845

Mann, D.T., Williams, A.M., Ward, P., \& Janelle, C.M. (2007). Perceptual-cognitive expertise in sport: A meta-analysis. Journal of Sport and Exercise Psychology, 29, 457-478. doi: 10.1123/jsep.29.4.457

Murray, N.P., \& Hunfalvay, M. (2017). A comparison of visual search strategies of elite and non-elite tennis players through cluster analysis. Journal of Sports Sciences, 35(3), 241-246. doi: 10.1080/02640414.2016.1161215

Newell, K.M. (1986). Constraints on the development of coordination. In M.G. Wade \& H.T.A. Whiting (Eds.), Motor development in children: Aspects of coordination and control (pp. 341-360). Dordrecht: Kluwer Academic Publishers Group.

Panchuk, D., \& Vickers, J.N. (2006). Gaze behaviors of goaltenders under spatial-temporal constraints. Human Movement Science, 25, 733-752. doi: 10.1016/j.humov.2006.07.001

Piras, A., Pierantozzi, E., \& Squatrito, S. (2014). Visual search strategy in judo fighters during the execution of the first grip. International Journal of Sports Science and Coaching, 9, 185-197. doi: 10.1260/1747-9541.9.1.185

Reina, R., Moreno, F.J., \& Sanz, D. (2007). Visual behavior and motor responses of novice and experienced wheelchair tennis players relative to the service return. Adapted Physical Activity Quarterly, 24, 254-271. doi: 10.1123/ apaq.24.3.254

Ripoll, H., Kerlirzin, Y., Stein, J.-F., \& Reine, B. (1995). Analysis of information processing, decision making, and visual strategies in complex problem solving sport situations. Human Movement Science, 14, 325-349. doi: 10.1016/0167-9457(95)00019-O

Singer, R.N., Williams, A.M., Frehlich, S.G., Janelle, C.M., Radlo, S.J., Barba, D.A., \& Bouchard, L.J. (1998). New frontiers in visual search: An exploratory study in live tennis situations. Research Quarterly for Exercise and Sport, 69, 290-296. doi: 10.1080/02701367.1998.10607696

Ward, P., Williams, A.M., \& Bennett, S.J. (2002). Visual search and biological motion perception in tennis. Research Quarterly for Exercise and Sport, 73, 107-112. doi: 10.1080/02701367.2002.10608997

Wilcox, R. (2017). Introduction to Robust Estimation and Hypothesis Testing. Ney York, NY: Academic Press.

Williams, A.M., Davids, K., Burwitz, L., \& Williams, J.G. (1994). Visual search strategies in experienced and inexperienced soccer players. Research Quarterly for Exercise and Sport, 65, 127-135. doi: 10.1080/02701367.1994.10607607

Williams, A.M., Davids, K., \& Williams, J.G. (1999). Visual perception and action in sport. New York, NY: E\&FN Spon.

Williams, A.M., \& Elliott, D. (1999). Anxiety, expertise, and visual search strategy in karate. Journal of Sport and Exercise Psychology, 21, 362-375. doi: 10.1123/jsep.21.4.362

Williams, A.M., Ward, P., Knowles, J.M., \& Smeeton, N.J. (2002). Anticipation skill in a real-world task: Measurement, training, and transfer in tennis. Journal of Experimental Psychology: Applied, 8, 259-270. doi: 10.1037/1076898X.8.4.259

Submitted: June 2, 2018

Accepted: May 10, 2021

Published Online First: December 3, 2021
Correspondence to:

Matheus Maia Pacheco, Ph.D.

Movement Control \& Neuroplasticity

Tervuursevest 101 - box 1501

KU Leuven

3001 Leuven, Belgium

Phone: +321632 4763

Email:mm16440@uga.edu 